\title{
Evolution physical intelligent guiding principle
}

\author{
Elsheikh M. Elsheik ${ }^{1}$ \\ ${ }^{1}$ University of Science and Technology, Omdurman, Khartoum, Sudan
}

Received: 12 November 2015/Revised: 20 December 2015/Accepted: 6 January 2016/Published online: 14 February 2016

(C) The Author(s) 2016. This article is published with open access at Springerlink.com

\begin{abstract}
Ordinary physics being unable to specify an intelligent guiding principle to account for the apparent life's intelligent design, some of the intelligent design movement advocates propose a metaphysical intelligent designer. In this regard, although intelligent design movement starts from a valid scientific premise, it ends up with a metaphysical inference that cannot be empirically falsified. Thus, it undermines its scientific credibility. Based on quantum information biology (QIB) which is a generalized physics hypothesis, we demonstrate that biological evolution is subject to a physical intelligent guiding principle (PIGP). Generalized physics (QIB) is a set of physical properties and laws that distinguish life from nonlife, irreducible to ordinary physics, and admit limiting transition to quantum mechanics. In other words, biology, or some aspects of it, is generalized physics. According to the PIGP, a species' increase in bio-complexity, phylogenetically, measured in terms of Jorgensen's eco-exergy density is a function of its bio-intelligence. Bio-intelligence has the dimensions of action, information and time; it is the capacity to generate bio-complexity and represents evolution target criterion. The PIGP does not clash with Darwinian evolution basic mechanism, random mutational changes and natural selection. Because natural selection selects beneficial mutations and beneficial mutations are those which satisfy the criteria of bio-intelligence, they are not random. Bio-intelligence is the origin of human intelligence, i.e., "The nature of intelligence is nature's intelligence."
\end{abstract}

Elsheikh M. Elsheik

elsheikh46@yahoo.com
Keywords Bio-information - Bio-intelligence · Eco-exergy $\cdot$ Evolution · Intelligent design, maximum action principle

\section{Introduction}

The causes of bio-information generation during ontogenetic growth and development, ecosystem growth and development, as well as during phylogenetic evolution, are one of the greatest challenges facing both theoretical physics and theoretical biology. Neo-Darwinism claims that such bio-information generation can be accounted for by natural selection acted upon random mutational changes. However, numerous scientists have questioned the efficacy of selection and random mutational changes as a mechanism for generating the bio-information necessary for morphological novelty (Eden 1966; Wadington 1968a, b, c; Gould 1982; Yockey 1992, Thomson 1992; Kauffman 1995; Perez 2010; Jorgensen 2007, 2012; Elsheikh 2010, 2014).

Eden who was especially concerned about the elements of randomness contended "No currently existing language can tolerate random changes in the symbol sequences which express its sentences. Meaning is almost invariably destroyed. Any changes must be syntactically lawful ones." Eden (1966). Whyte (1965) suggested that in addition to Darwinian selection there should be an internal selection of mutants at the molecular, chromosomal and cellular levels, in accordance with their compatibility with internal coordination of the system. Waddington (Waddington 1968a, b, c) tried to show that evolution does not depend on random search. He emphasized that what occurs randomly are the mutations on the genome level; 
however, the output of these changes on the phenotype is not random, i.e., there are certain operators that map the space of genotypes into a "fitness space." Dawkins (1986) proposed what he called cumulative selection as an alternative to what he called single-step selection.

Based on his proposed Ecological Law of Thermodynamics (ELT) which states "A system that receives a throughflow of exergy (high-quality energy) will try to utilize the exergy flow to move away from thermodynamic equilibrium, and if more combinations of components and processes are offered to utilize the exergy flow, the system will select the organization that gives the system as much exergy content (storage) as possible, i.e., maximizes $\mathrm{dEx} /$ dt.," Jorgensen $(2006,2007,2012,2015)$ demonstrates that biological evolution maximizes eco-exergy density. Ecoexergy is an organism's work energy, including the information work energy embodied in the genes; it could also be understood as the organism bio-mass and the genetic information embedded in this mass.

McClendon (1980) compared biological evolution with chemical evolution of isotopes. From the comparison, he concluded that the forces which drive biological evolution are intrinsic property of matter. In other words, the evolution of novel, more complex organisms, from lower ones precedes adaptation and selection. Kauffman (1995) had a similar view. He suggests that selection acts, not only on random variations, but also on emergent patterns of order that self-organize via the laws of nature. Perez (2010) demonstrated that there is an evolutionary matrix that governs the structure of DNA, so that beneficial mutations cannot be random. Davies $\mathrm{P}$ is concerned with whether there are nontrivial quantum phenomena relevant for biology. Nontrivial is meant the presence of long-ranged, long-lived, or multiparticle quantum coherences, the explicit use of quantum entanglement, etc. He emphasized "If quantum mechanics is to play a nontrivial role in biosystems, then some way to sustain quantum coherence at least for biochemically, if not biologically, significant time scales must be found. Without this crucial step, quantum biology is dead" Davies (2004). Pattee (1968) asserts that if living matter is exactly the same as nonliving matter with respect to description by physical laws, then this does not answer the obvious question of why living matter is so conspicuously different from nonliving matter.

Certain authors regard these inadequacies and conflicting hypotheses as a decisive refutation of Darwinism and hence call for an intelligent designer (Meyer 2004; Dembski 1998). Truman (1999) challenged naturalists to demonstrate where does information come from in the first place and, secondly, how could it increase over time. Dembski asserts "Natural causes are in principle incapable of explaining the origin of complex specified information
(CSI). To be sure, natural causes can explain the flow of CSI, being ideally suited for transmitting already existing information. What natural causes could not do, however, is originate CSI. This strong proscriptive claim, that natural causes can only transmit CSI but never originate it, I call the law of conservation of information. It is this law that gives definite scientific content to the claim that CSI is intelligently caused" Dembski (1998).

Although intelligent design movement starts from a valid scientific premise concerning the bio-systems intelligent design, it ends up with a metaphysical inference that cannot be empirically falsified. Thus, it undermines its scientific credibility. Moreover, the notion of complex specified information CSI advocated by intelligent design movement is not operationally defined in order to prove that information cannot originate or increase by naturalistic causes. Under such circumstances, any quantification of bio-information (i.e., CSI) and the discovery of the laws of its increase, as we would like to demonstrate, will save the efforts to invite god of the gaps.

To overcome these difficulties concerning the nature of life and its evolution, Elsheikh $(2010,2014,2015)$ proposes broadening the ontological foundation of contemporary physical theory, by giving answers to the following questions:

- What is the physical property that distinguishes life from nonlife and contains the dynamical essence of living systems? The physical property that plays in biology the unifying role the concept of energy plays in physics?

- What is the physical law or principle associated with the above-mentioned property and in consequence itself must distinguish life from nonlife?

- What is the field material substrate that embodies the mentioned property and its associated principle?

- What are the field equations and how can they describe biotic evolution and development.

- How can they reveal the evolution physical intelligent guiding principle?

\section{Methods}

\subsection{Quantum information fractal field hypothesis (QIFFH)}

Elsheikh $(2010,2014,2015)$ proposes the hypothesis of QIB. QIB is the study of biosystems as spontaneous selforganizing dynamical systems. QIB bridges the gap between physics and biology and proposes a unified theory of life according to which both phylogeny and ontogeny 
can be studied on the basis of QIFF equations. To accomplish this goal, Elsheikh proposes broadening the concepts and principles of information, least action principle and quantum field.

\subsection{Bio-information}

$\mathrm{He}$ distinguishes between genome physical information which is a measure of genome static physical complexity in bytes, and genome's bio-information a measure of genome's bio-complexity which is developmental and functional, and has the dimensions of energy and information. In this new perspective, the genome's bio-information $(v(t))$ is about the phenotype, since the genome's bio-information is meaningless without producing a phenotype. The bio-information increases before adulthood, has a maximum when the organism is fully grown, decreases afterward and becomes zero when the organism dies. For example, considering a unicellular organism that divides for successive generations, the bio-information becomes a periodic function of time. Thus, it represents the bio-information oscillations generated by the genome through successive generations.

\subsection{Least action principle}

In physics, the principle of least action, or more accurately the principle of stationary action is a variation principle when applied to the action of a mechanical system, can be used to obtain the equation of motion for that system. According to the least action principle, a particle moves along the path for which the action is minimum; this means the spontaneous motion of the particle is to minimize action. Now is it possible to extend the action principle to incorporate the case of maximum action? Grandpierre (2007). In general, a maximum or most action principle must allow a system to follow spontaneously a path of maximum action. Thus, spontaneous self-organization becomes possible, e.g., embryogenesis and morphogenesis, because under such circumstances the maximum action principle maximizes the rate of change of action, whereas under the least action principle the rate of change of action is less or equals zero.

\subsection{DNA fractal nature}

Dan Winter-a pioneer on golden ratio in physics-asserts that golden ratio fractality is a condition of recursive constructive interference. In his view, DNA golden ratiobased dodecahedron fractal geometry is the only geometry that allows wave patterns to add and multiply recursively constructively, thus producing a vortex for imploding charge waves along phi-spiral paths which are paths of maximum action and maximum coherence. He coined the term quantum fractal field to designate the state of perfected charge distribution and coherence characteristic of the DNA. Dan Winter (2012).

\subsection{Quantum field}

A field whether classical or quantum is defined as a function over space and time. This definition is not sufficient to contain the dynamical essence of biosystems. Because a biosystem dynamics or functionality depends on its bioinformation or bio-complexity rather than on the space coordinates it occupies. So, Elsheikh defines the genome quantum information fractal field (QIFF) as a function over bio-information and time, $\mathrm{L}(\mathrm{v}, \mathrm{t})$. QIFF is the union of DNA golden ratio-based fractal geometry and the maximum action principle. Such field generates, in addition to weak EM waves, self-sustained bio-information oscillations for successive generations. This means the DNA or genome is the material substrate of the QIFF.

Definition 1 A genome or genome pool is a quantum information fractal field, QIFF.

\subsection{Postulates}

1. The QIFF (genome) generates, in addition to weak EM waves, self-sustained bio-information oscillations through successive generations

2. The bio-information oscillations contain the dynamical essence of the living system.

3. The bio-information sustains the living state.

Definition 2 Bio-information which is the information stored in DNA and proteins is developmental functional complexity, within a specific environmental context.

Definition 3 Vitality, $v(t)$, is the genome capacity to generate developmental functional complexity (bio-information), i.e., the capacity to generate phenotype, where (t) is the time measured from the moment of initial growth.

Based on these postulates and definitions, Elsheikh (2010, 2014, 2015) defined genome's bio-information (developmental functional complexity) in terms of vitality, $v(t)$, function:

$v(t)=b E(t) \ell^{a}$

where $\mathrm{b}$ is genome's physical information measured in bytes (Adami 2002), $E$ ( $t$ ) organism's total energy metabolized, $\ell=\mathrm{A}-\mathrm{t}$ is the organism life expectancy, A lifespan or cell cycle time and $a$ is an exponent which depends on species. 
Vitality satisfies the following property:

It increases before adulthood, reaches a maximum at adulthood, decreases afterward and becomes zero when the organism dies, i.e., $v(A)=0$.

The vitality model may be usefully employed to discuss vitality for successive generations. We shall essentially be concerned with unicellular organisms, particularly those which reproduce by binary fission. For such systems, we suggest the following equation:

$v_{g}=v(t+m A)=v(t)$

where $m=1,2,3, .$. , is the number of cell divisions or generations. Equation (2) defines vitality as a periodic function of time, i.e., it represents vitality oscillations, or equally acceptable, the bio-information oscillations generated by the genome as self-replicating quantum information fractal field. Consequently, the genome's bioinformation measured in calories $\mathrm{x}$ bits, oscillates in the time-vitality ( $\mathrm{t}-\mathrm{v})$ space, also called bio-information space, during successive generations. This model is equally applicable to multi-cellular organisms which reveal an overlap of generations, i.e., overlap of bio-information oscillations (Fig. 1).

\subsection{The Bio-information attractor}

Based on the first and second postulates of QIFFH, the life state of an organism, L, called the bio-information attractor (or the life-organizing principle) being an attribute of QIFF, must satisfy the following conditions:

1. $L=\mathrm{L}(\mathrm{v}, \mathrm{t})$

2. $L$ is a generalized Schrödinger's type of system.

3. $L$ is a periodic functional attractor.

We shall therefore assume the simple following form:

$L=L_{0} \mathrm{e}^{\mathrm{i} \Phi / \mathrm{G}}$

where $L_{0}$ is the amplitude and $\mathrm{G}$ is a constant to be found and has the dimension of Planck's constant. To satisfy the

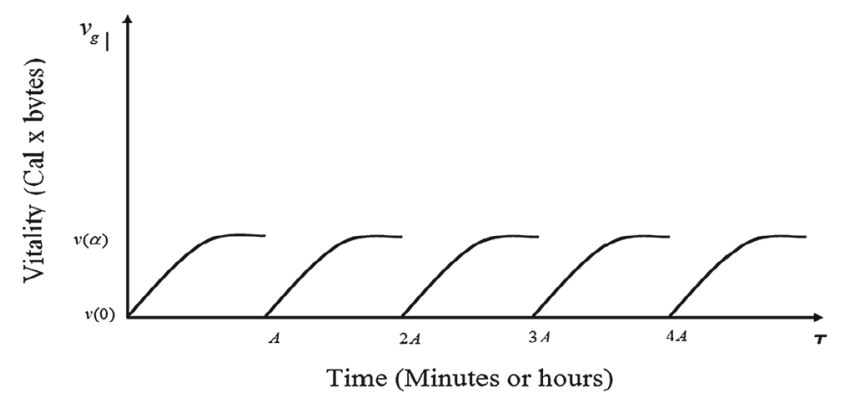

Fig. 1 Hypothetical representation of bio-information oscillations of a unicellular organism for successive generations. $A$, the lifespan, is the period of oscillations (Elsheikh 2015) above-mentioned conditions, we limit our considerations to the concrete example in which $\Phi$ is given by:

$\Phi(t)=\int_{0}^{t} E\left(1-\frac{x}{A}\right)^{a} \mathrm{~d} x$

$\Phi(t)=\frac{V(t)}{b A^{a}}=\frac{1}{b A^{a}} \int_{0}^{t} v(x) \mathrm{d} x$

From (3), (4) and (5), we get:

$\ddot{L}-\frac{\dot{v}}{v} \dot{L}+\frac{v^{t 2}}{k^{2}} L=0$

where $k=b A^{\mathrm{a}} \mathrm{G}$.

Elsheikh shows that Eq. (6) represents a nonlinear, nonconservative and irreversible system, which describes self-sustained oscillations (2010, 2014, 2015). Furthermore, it has been proved that Eq. (6), being a generalized Lienard's system, admits limit cycle. Stable limit cycle solutions usually characterize structural stability or dynamic equilibrium, a property of high significance to bio-systems (Minorsky 1962, Nicolis and Prirogine 1977, Goodwin 1985). A limit cycle is also called an attractor, i.e., a set of states of a dynamic physical system toward which that system tends to evolve, regardless of the initial conditions of the system. We call the bio-information attractor that describes muti-cellular organism dynamics a major attractor, while that which describes cellular dynamics a minor attractor. A cell type is an example of a minor attractor which belongs to the basin of a major attractor.

Elsheikh (2010, 2014), assuming evolution (mutation, selection, etc) a process through which the bio-information attractor undertakes negative damping, proves that evolution leads to the increase or maximization of total vitality which is the area under the vitality curve, $V(A)$. Although the genome is the source for generating the bio-information attractor, the bio-information attractor by describing the dynamics of cellular and multi-cellular bio-systems contains the genome as subsystem or sub-attractor that belongs to the basin of the bio-information attractor. This situation creates genome-phenome reciprocal causality, such that a bio-system is subject both to upward and downward causation.

\subsection{Field equations}

In addition to the periodic bio-information attractor:

$\ddot{L}-\frac{\dot{v}}{v} \dot{L}+\frac{v^{2}}{k^{2}} L=0$

Elsheikh $(2014,2015)$ derived the following laws: 


\subsection{First law of self-organization}

To account for the spontaneous growth, development and functional activity of living systems, the living system must maintain a path of maximum action. Under such circumstances, the genome capacity to generate developmental functional complexity (vitality) must be correlated with the rate of change of action to match the path. Thus, we demonstrate that the phase of the genome's bio-information oscillations, which has action units, identifies the path of maximum action we are looking for, from (5) we get:

$\therefore \dot{\Phi}(t)=K v(t)$

where $K=b^{-1} A^{-a}$.

Equation (7) establishes the correspondence of energy and bio-information along the time domain.

From (7):

$\therefore V(t)=b A^{a} \Phi(t)$

$\therefore V(A)=b A^{a} \Phi(A)$

where $V(A)$ is total vitality, given by:

$V(A)=\int_{0}^{A} v(t) \mathrm{dt}$

By definition, total vitality represents the genome or QIFF capacity to generate bio-complexity. However, the biocomplexity of different species is given by:

$v_{s}(\alpha)=A^{-a} v(\alpha)=A^{-a} b E(\alpha) \ell^{a}(\alpha)=\dot{\Phi}(\alpha) b$

$\alpha$ is the time when the organism is fully grown.

\subsection{Second law of self-organization}

It is also significant to show that biological information (total vitality) is also quantized. Such quantization has its expression in beneficial mutational changes which uncover the genome stable or quasi-stable states. For this purpose, the bio-information attractor (the life-organizing principle) could be employed to derive the proposed quantization relationship. The derived quantization relation may provide plausible theoretical basis for punctuated equilibrium. Thus, given (3) and (5) we get:

$L(t)=L_{0} e^{\frac{i V(t)}{k}}=C \cos \frac{V(t)}{k}+B \sin \frac{V(t)}{k}$

Setting the boundary conditions:

At $t=0$, biotic irreversibility (represented by the age of the organism, (Elsheikh 2015) is zero, so $V(0)=0$, we let $L(0)=0$.
At $t=A$, the system is dead and $V(A)=0$, and we let $L$ (A) $=0$.

$\therefore 0 \leq t \leq A$

This means the life of an organism is bounded, i.e., closed within the interval (12), and thus, the organism has no life before birth and has no life after death.

From (11) and the boundary conditions, we get:

$$
\begin{aligned}
& C=0, \frac{V(A)}{k}=n \pi, \quad n=1,2, . . \\
& \begin{aligned}
V(A) & =n \pi k=n \pi b G A^{a} \\
& =\frac{n \pi b G}{f^{a}}
\end{aligned}
\end{aligned}
$$

Note the lifespan, $A$, is at the same time the period of oscillations. Equation (13) is the second law of self-organization; it is a quantum information fractal law of evolution and development.

\subsection{Law of conservation of total bio-information}

$T=U(t)+Z(N(t))=$ constant

$U(t)$ is the population mean total vitality at time t during successive generations, and $Z(N(t))$ is total natality density function at time $\mathrm{t}$ during successive generations. Elsheikh (2014, 2015) shows that Eq. (14) can be employed to derive logistic equations for the growth and development of organisms and populations:

$\therefore \quad \frac{d p}{d t}=p\left(c-\int_{0}^{t} \frac{\dot{U} d x}{Z_{N}^{\prime}}\right)$

where $\mathrm{p}$ is population size.

Moreover, to substantiate the notion of QIFF, Elsheikh (2015) demonstrates that cell type, tissue, organ, organ system and organism represent a nested hierarchy of bioinformation attractors, i.e., nested hierarchy of quantum information stationary functional states whose maximum size is given by (15).

\section{Results}

\subsection{Evolution physical intelligent guiding principle (EPIGP)}

We have got two measures vitality as a measure of biocomplexity given by Eq. (10) and total vitality as a measure of the genome's capacity to generate bio-complexity given by Eq. (8). Now the capacity to generate bio-complexity could also be an appropriate definition for bio- 
intelligence; in consequence, the terms total vitality and bio-intelligence can be used interchangeably.

Note for a given species, under constant environmental conditions, $V(A)$ remains constant for successive generations, in accordance with the following theorem:

$V(A)=\int_{0}^{A} v(t) \mathrm{dt}=$ constant

Proof Given: $V(t)=\int_{0}^{t} v(x) \mathrm{dx}$

$\therefore \dot{V}(t)=v(t)$

$\therefore \dot{V}(A)=v(A)=0$

$\therefore V(A)=$ constant,

This is why a species preserves its kind through successive generations indefinitely under constant environmental conditions.

On the other hand, $V(A)$ for different species due to phylogenetic evolution changes, so let phylogenetically $V$ $(A)=B_{i}$, where $B_{i}$ is bio-intelligence; then from (8), we get:

$B_{i}=\Phi(A) b A^{a}$

Definition Bio-intelligence, $B_{i}$, is the capacity to generate bio-complexity, or functional structures.

Thus, bio-intelligence has the dimensions of action, information and time. It is the genome's capacity to generate bio-complexity, or functional structures, and represents the evolution target criterion. To substantiate this view, we employ Eq. (10):

$v_{s}(\alpha)=A^{-a} v(\alpha)=A^{-a} b E(\alpha) \ell^{a}(\alpha)=\dot{\Phi}(\alpha) b$

where $E(\alpha)$ is total energy metabolized from the moment of initial growth to time $\alpha$ when the organism is fully grown, and $\ell(\alpha)$ is the organism life expectancy at time $\alpha$,

$\dot{\Phi}(\alpha)$ is the rate of change of action at time $\alpha$ :

$\dot{\Phi}(\alpha)=E(\alpha)\left(1-\frac{\alpha}{A}\right)^{a}$

It is clear from (10) the increase in $v_{s}(\alpha)$ necessitates the increase in $\dot{\Phi}(\alpha)$ and $b$, genome physical information, (Sharov 2006; Marcov et al. 2010). However, the increase in $\dot{\Phi}(\alpha)$, from (18), necessitates the increase in $E(\alpha)$ which in turn requires the following conditions:

1. Maximization of $\alpha$ so the organism metabolizes more energy.

2. Minimization of $\frac{\alpha}{A}$.

To satisfy both conditions forces the increase in A, the organism lifespan. Since the increase in $v_{s}(\alpha)$ involves the increase in $\mathrm{b}, \dot{\Phi}(\alpha)$, and $\mathrm{A}$ which at the same time contribute to the defining parameters of $B_{i}$, it follows we may reasonably assume that, using (10) and (17), in general bio-complexity is a function of bio-intelligence:

$v_{s}(\alpha)=F\left(B_{i}\right)$

In particular, if nature becomes generous to show simplicity, we may have:

$v_{s}(\alpha)=c_{1} B_{i}$

where $c_{1}$ is proportionality constant. We denote Eq. (20) the evolution physical intelligent guiding principle (EPIGP).

$B_{i}$, being the capacity to generate species bio-complexity, defines the direction of evolutionary progress, i.e., defines the evolution target criterion. Evolutionists used to measure body size, genome physical information or lifespan in order to identify evolutionary progress, but in each case, they found exceptions. Now bio-intelligence asserts that evolutionary progress is the product of all these factors or parameters taken together. The more evolved species is the one having greater bio-intelligence, i.e., greater fusion of action, information and time. The EPIGP does not clash with Darwinian evolution basic mechanism, random mutational changes and natural selection. Because natural selection selects beneficial mutations and beneficial mutations are those which satisfy the criteria of bio-intelligence.

Moreover, Jorgensen $(2007,2012,2015)$ defines ecoexergy density $\left(E_{x}\right)$ by:

$E_{x}=\beta \times 18.7 \frac{\mathrm{kJ}}{\mathrm{g}}$

$\beta$ is a weighting factor based on information. It is clear although eco-exergy density has the dimensions of energy; the definition involves the product of the organism's embedded genetic information and its work energy per unit of biomass. So there is strong correlation with $v_{s}(\alpha)$. In fact, Elsheikh (2014) discussed the correspondence between vs $(\alpha)$ and eco-exergy density or exergy storage and, in consequence, a correspondence between Jorgensen's Ecological Law of Thermodynamics and the maximum action principle, so it is reasonable to correlate $E_{x}$ to bio-intelligence:

$E_{x}=c_{2} B_{i}$

where $c_{2}$ is proportionality constant. In this case, fortunately, Jorgensen has prepared the experimental data for $E_{x}$, where he demonstrates that evolution maximizes ecoexergy density, producing a plot that has some approximation to exponential growth. Elsheikh $(2014,2015)$ argues that the plot could represent good approximation to power law distribution similar to the one given by Elsheikh's proposed second law of self-organization. The second law shows that evolution maximizes bio-intelligence inversely proportional to the frequency of bio- 
information oscillations. Elsheikh also argued that exponential growth arises when we deal with physical information measured in bytes as in the case of Moore's law. However, bio-complexity, as defined by Jorgensen, Ulanowicz, Elsheikh and may be others, has the dimensions of energy and information and produces power law distribution, which can also account for Cambrian explosion. Thus, bio-intelligence, as power law distribution, driven by the maximum action principle, squeezes the evolution timescale into the lifespan of the Earth (Fig. 2).

\section{Courtesy of Sven Eric Jorgensen}

\subsection{Definition of life}

Life must satisfy the maximum action principle and bioinformation which distinguish it from nonlife. It follows:

Life is spontaneous self-organization bio-information phenomenon. This can be put in the following mathematical form:

A system is living if:

$\ddot{\Phi}(0)>0, \quad$ and $\quad \dot{v}(0)>0$

A system is nonliving if:

$\ddot{\Phi}(0)=0, \quad$ and $\quad \dot{v}(0)=0$

It is clear a system is nonliving if its total energy remains constant and $\dot{v}(0)=0$.

\subsection{Physical origin of bio-information}

The origin of DNA and its genetic code is a deterministic consequence of the union of the maximum action principle

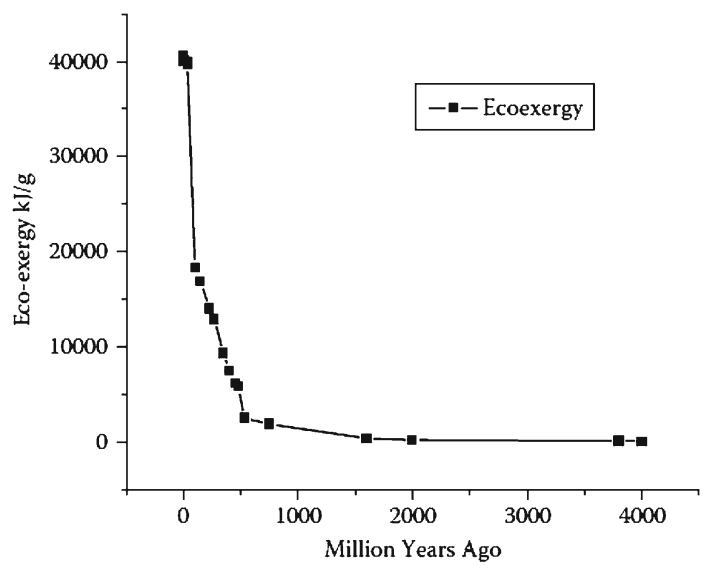

Fig. 2 Eco-exergy density in $\mathrm{kj} / \mathrm{g}$ is plotted versus the time. Notice the abrupt increase at the beginning of the Cambrian time, about 540 million years ago. Also the enhanced increase by emergence of mammals, hominoids and humans can be seen clearly on the graph. See also Jorgensen (2012) and the DNA's golden ratio-based dodecahedron fractal geometry. Thus, origination of bio-information necessitates the following conditions:

1. Sequential arrangement of DNA nucleotides along a path of maximum action. This condition is satisfied by the DNA helical and golden ratio-based fractal geometry.

2. Sequential arrangement of DNA nucleotides along a path of maximum bio-information. This condition necessitates assigning bio-information (energy $\mathrm{x}$ information) weights to the DNA different nucleotides in order to be arranged into words (codons), sentences and texts (genes) of maximum bio-information, hence manifesting the bio-information code embedded and hidden inside the standard genetic code. The embedded bio-information code is contextually richer than the standard genetic code, because it contains homonyms (a homonym is a word that has more than one meaning). Based on such bio-information code, a gene becomes contextually rich, i.e., capable of producing more than one protein.

Thus, the physical origin of bio-information resides in the sequential arrangement of DNA nucleotides along paths of maximum action and maximum bio-information. Moreover, on one hand, the first law of self-organization increases bio-information ontogenetically, and on the other hand, the physical intelligent guiding principle increases bio-information phylogenetically.

\subsection{Quantization of bio-intelligence}

Applying the second law to phylogenetic evolution yields:

$B_{i}=\frac{n \pi b G}{f^{a}}$

This law demonstrates that bio-intelligence is quantized and bifurcates in accordance with Fibonacci numbers $(1,2$, $3,5,8,13 \ldots)$ to create specific disjoint stationary functional states.

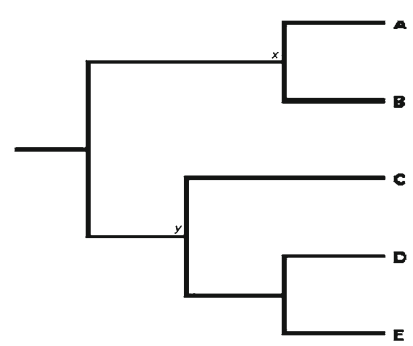

Moreover, it is a power law, a Pareto (1897) type of law, which indicates that bio-intelligence varies directly with Fibonacci numbers and genome physical information and 
inversely with the frequency of bio-information oscillations. It is interesting that Bak and Paczuski (1995) have also proposed a power law to account for punctuated equilibrium, Eldredge and Gould (1972). However, based on (25) the motif of bifurcations occurs in accordance with Fibonacci numbers, n. In consequence, (25) generates treelike structures ontogenetically as well as phylogenetically. Such result can be empirically underpinned by development ontology tree, Edgar (2013) and the tree of life, Rosindell (2012).

Elsheikh $(2010,2014,2015)$ indicates that speciation is a punctuationistic transition from a lower major bio-information attractor to an upper major attractor, governed by the second law of self-organization. Consequently, the existence of disjoint stationary states (attractors) implies that transitional forms are genomically unstable, transient dwellers, which may explain the absence or scarcity of their fossil records. The bio-information attractor which represents the organism as a whole is a major attractor with respect to Kauffman (1995) ontogenetic attractors which represent the different cell types during the organism development.

As stated above, the second law of self-organization, Eq. (25), is a power law, i.e., scale invariant, and it describes the development and evolution of cellular organisms and multi-cellular organisms, reflecting the self-similarity of biological hierarchy. The law has also an important aspect of quantization, which can be revealed by inquiring about the nature of the integer $n$. The integer $n$ is supposed to account for the functional stationary quantum states of a living system, and the stability and functionality of its pattern formations. For this sake, let us suppose an organism having total action $\Phi_{1}$ produces another organism of total action $\Phi_{2}$; in consequence, three possibilities emerge:

Case $\Phi_{2}=\Phi_{1}$, in this case the organism $\Phi_{1}$ is said to 1 be stable or in stationary state

Case $\Phi_{2}<\Phi_{1}$, in this case the organism $\Phi_{1}$ is said to

2 be degraded, and it is a path preferable by the least action principle and the second law of thermodynamics

Case $\quad \Phi_{2}>\Phi_{1}$, in this case the organism $\Phi_{1}$ is said to 3 grow or evolve, i.e., manifests the maximum action principle. However, to accommodate the maximum action principle and the scale invariance of the second law, we consider the following conditions:

1. $\Phi_{2}>\Phi_{1} \Rightarrow \frac{\Phi_{2}}{\Phi_{1}}>1$

2. $\frac{\Phi_{2}}{\Phi_{1}}=\frac{\Phi_{1}+\Phi_{2}}{\Phi_{2}}$

Condition 2 preserves the scale invariance. Restating the above conditions in terms of $n_{1}$ and $n_{2}$ which are real numbers associated with $\Phi_{1}$ and $\Phi_{2}$ such that $n_{2}>n_{1}$, we get:

$\frac{n_{2}}{n_{1}}=\frac{n_{1}+n_{2}}{n_{2}}=\frac{n_{1}}{n_{2}}+1$ let $\frac{n_{2}}{n_{1}}=x$ we get:

$x=\frac{1}{x}+1 \quad \therefore x^{2}-x-1=0$

$\therefore x=\frac{n_{2}}{n_{1}}=\frac{1+\sqrt{1+4}}{2}=1.618 \cdots=$ golden ratio

This means the golden ratio, being widespread in living systems, is a deterministic consequence of the maximum action principle and fractality, Mandelbrot (1982), and that $\mathrm{n}$ in the fractal law, Eq. (25), is Fibonacci's number.

Based on the second law of self-organization, both phylogeny and ontogeny are processes that generate and assemble minor attractors associated with Fibonacci numbers. In consequence, it is reasonable to assume that the tree of life as well as ontogenetic cellular differentiation could be subject to computer simulation on the basis of the field equations. The realization that phylogenetic evolution (macroevolution) is driven by a power law distribution (the second law) may shed some light on the Cambrian explosion. Accordingly, $80 \%$ of the initial time of phylogenetic evolution contributes $20 \%$ of bio-intelligence increase, whereas the $20 \%$ of the succeeding time contributes $80 \%$ of bio-intelligence increase, which may account for the Cambrian explosion. Now, since the Cambrian explosion started approximately 0.6 billion years ago, it means that life originated approximately 3 billion years ago.

\subsection{Conservation of total bio-information}

In addition, Eq. (14) can also be used to describe phylogenetic evolution. For example, since the evolutionary process, in general, maximizes bio-intelligence, based on (14) evolution also minimizes natality rate. This resolves Waddington's problem "For us the major problem is one which was only a second order issue for Darwin. This is the problem of adaptation. Why do we find animals and plants which have structures and capacities that make them admirably suited to carry out extraordinary living routines in the most unlikely situations, often highly unfavorable for reproduction?" (Waddington 1968a, b, c). Furthermore, if a population or species is somehow forced to regress, i.e., its bio-intelligence decreases, then from (14), its natality rate must increase. Otherwise, the species is susceptible to extinction.

\subsection{Multi-level selection}

One of the important discoveries of QIB is that the genome total bio-information generates two survival components: 
reproductive fitness component and bio-intelligence fitness component. Then, since phylogenetic evolution maximizes bio-intelligence, it follows conservation of genome total bio-information which leads to the decrease in natality rate, i.e., decreases the number of offspring propagated and the mechanisms supporting this fitness, such as selfishness and violence. This process is in favor of consolidating altruism, which is quite evident from the evolution of major transitions. The major transition in evolution refer to the transitions from solitary replicators to network of replicators enclosed within compartments, from independent genes to chromosomes, from prokaryotic cells to eukaryotic cells containing organelles, from unicellular to multicellular organisms and from solitary organisms to colonies, (Okasha 2005).

Wilson and Wilson (2007) emphasize that there is agreement that selection occurs within and among groups, that the balance between levels of selection can itself evolve and that a major transition occurs when selection within groups is suppressed, enabling selection among groups to dominate the final vector of evolutionary change.

Bio-intelligence, by maximizing both creativity and altruism, facilitates the group selection fitness unit, in support of Wilson and Wilson (2007) proposed sociobiology's new theoretical foundation: "Selfishness beats altruism within groups. Altruistic groups beat selfish groups. Everything else is commentary.” Thus, extending new Darwinian theory by accommodating a new fitness component substantiates the theory of multi-level selection by identifying the group selection fitness unit.

\subsection{Origin of human intelligence}

According to the EPIGP, production of new bio-intelligence is phylogenetic property. We assume that due to the evolution of Homo sapiens brain architecture and the emergence of human higher mental powers, bio-intelligence as capacity to generate functional structures has been realized as an ontogenetic property. Thus, humans become both products and producers of bio-intelligence. Humans, being producers of bio-intelligence, signify, originate the socio-cultural evolution and, at the same time, explain why human socio-cultural evolution maximizes bio-intelligence faster than genetic evolution.

Human intelligence as the ability to acquire and apply knowledge creates nonmaterial functional structures, e.g., language, mathematics, philosophy, arts and material functional structures, e.g., cars, factories, aero planes and computers. These functional structures neither reproduce nor evolve spontaneously, so human intelligence is still far behind life's bio-intelligence. Nonetheless, it is evident that "The nature of intelligence is nature's intelligence."

\section{Discussion}

Is it possible within the domain of present-day standard physics to claim that life has a physical intelligent guiding principle? The answer is big no. Why? Because revealing the PIGP necessitates the extension of the domain of standard physics in order to incorporate life phenomenon. It is highly significant, within the extended domain, to look for a working hypothesis, a unifying concept that plays in biology the role the concept of energy plays in physics. Energy is not appropriate to contain the dynamical essence of bio-systems, because bio-systems are also information processors. Information also is inappropriate not only because a bio-system is a system of energy but also because Shannon type of information, being a measure of improbability, measures static complexity, while biocomplexity is developmental and functional. Thus, the search for the unifying concept of biology necessitates a clue; the clue is to look for a physical property that distinguishes life from nonlife.

We suggest that bio-information which is a fusion of energy and information and which becomes zero for inanimate systems is the required unifying concept of biology. We proved that bio-information like energy is conserved and quantized, but unlike energy it self-organizes, because while inanimate systems are subject to the least action principle, bio-systems are subject to the maximum action principle. The maximum action principle, being the physical law that distinguishes life from nonlife, complies with and underpins bio-information. In consequence, both phylogeny and ontogeny are subject to the maximum action principle, i.e., to the field equations of QIB.

Probably, some physicists may worry about the fate of the least action principle. I urge them to be comfortable because the maximum action principle does not violate the least action principle. The situation is similar to the relationship between relativity or quantum mechanics and classical mechanics; relativity does not violate classical mechanics rather it covers a domain which is beyond the domain of validity of classical mechanics. And since the new domain of relativity is more general, it contains the laws of classical mechanics as special case. It is same with the maximum action principle which operates beyond the domain of the least action principle, and contains the least action principle as special case.

Consider the first law of self-organization [Eq. (7)] when $t=A$, i.e., an organism is dead, the rate of change of action becomes zero which means the inanimate system becomes in stationary state; moreover, the second time derivative is also zero which indicates that the system's total energy is conserved. More importantly, we, as 
scientists, must stick to empirical evidence as it is clear a bio-system, ontogenetically (embryogenesis and morphogenesis), as well as phylogenetically, traces a path of maximum action. Without the maximum action principle, I emphasize, with full confidence, that physics will remain forever blind to comprehend life phenomenon.

In fact, Davies (2004) asserts that life being an emergent phenomenon exhibiting novel properties and principles is not in conflict with causal closure at the microscopic level. He argues that advances in cosmological theory suggesting an upper bound on the information processing capacity of the universe (Lloyd 2002) may resolve this conflict for systems exceeding a certain threshold of complexity. A numerical estimate of the threshold places it at the level of a small protein. He indicates that this result may be traced in part to the operation of as-yet-tobe-elucidated biological organizing principles, consistent with, but not reducible to, the laws of physics operating at the microlevel. I call such organizing principle the life-organizing principle or the bio-information attractor.

Finally, the bio-information attractor is a minor attractor when describing cellular dynamics and a major bio-information attractor when describing multicellular organism dynamics. A cell type is an example of minor bio-information attractor which belongs to the basin of a major bioinformation attractor. Thus, both phylogeny and ontogeny are processes of generation and assembly of minor attractors. The attractors' quantum information stationary functional states are given by Fibonacci numbers. Although mutational changes are random, beneficial mutations for which the bio-information attractor undertakes negative damping are beneficial because they generate or consolidate a bio-intelligence quantum information stationary functional state, so they are not random.

\section{Conclusion}

To reveal the evolution physical intelligent guiding principle (EPIGP), it was necessary in the first place to distinguish between genome's physical information and genome's bio-information. The genome's bio-information is about the phenotype, it is a measure of developmental functional complexity, and it increases before adulthood, has a maximum when an organism is fully grown, decreases afterward and becomes zero when the organism dies. Thus, it distinguishes life from nonlife and has the dimensions of energy and information because there is no function without energy. Vitality, being a measure of the genome's capacity to generate bio-complexity, is mathematically defined in order to satisfy the above-mentioned bio-information criteria. So while vitality represents biocomplexity, total vitality represents the genome's capacity to generate bio-complexity, i.e., represents bio-intelligence.
Since physical properties are usually underpinned by physical laws, the spontaneous increase in bio-information before adulthood must be underpinned by a physical law that complies with it, so the law or principle itself must distinguish life from nonlife. The best candidate in this regard is the maximum action principle. Thus, it is proved that a bio-system's rate of change of action is directly proportional to the increase in its bio-information.

DNA is information storage, processor and replicator; in addition, due to its helical structure and golden ratio-based fractal geometry, it becomes a vortex for charge-wave implosion that maintains maximum coherence. Hence, it embodies the maximum action principle and generates bioinformation oscillations for successive generations. It resembles the material substrate for a quantum information fractal field (QIFF). The QIFF is a function over bio-information and time; it is a nested hierarchy of bio-information attractors toward which a bio-system is attracted.

The life state of an organism, being an attribute of a QIFF, is represented by a periodic bio-information functional attractor which is a generalized Schrodinger type of system. Based on the bio-information attractor, the other field equations are derived, and these are the first and second laws of self-organization.

Now we have a measure of bio-complexity for different species, and a measure for their capacity to generate biocomplexity which we call bio-intelligence; therefore, it is reasonable to assume that the specie's bio-complexity is a function of its bio-intelligence. In consequence, biological evolution, underpinned by the maximum action principle, maximizes bio-intelligence. On this perspective, Darwinian theory becomes more intelligible, because as natural selection provides the mechanism, the maximum action principle provides the driving force. It follows both questions of how and why evolution occurs are answered on naturalistic basis.

Acknowledgments I'm grateful to Professor Sven Eric Jorgensen for kind help and support.

Open Access This article is distributed under the terms of the Creative Commons Attribution 4.0 International License (http://crea tivecommons.org/licenses/by/4.0/), which permits unrestricted use, distribution, and reproduction in any medium, provided you give appropriate credit to the original author(s) and the source, provide a link to the Creative Commons license, and indicate if changes were made.

\section{References}

Adami C (2002) What is Complexity? BioEssays 24:1085-1094 Bak P, Paczuski M (1995) Complexity, contingency, and criticality. Proc Natl Acad Sci USA 92:6689-6696

Davies PCW (2004) Does quantum mechanics play a non-trivial role in life? BioSystems 78(2004):69-79 
Dawkins R (1986) The Blind Watchmaker. Longman Scientific and Technical. Longman Group, UK

Dembski W (1998) The design inference. Cambridge University Press, Cambridge

Eden M (1966) Inadequacies of New Darwinian evolution as a scientific theory. In: Moorhead SP, Kaplan MM (eds) Mathematical challenges to the new-Darwinian interpretation of evolution. The Wistar Institute Press, USA

Edgar R (2013) The embryonic development, stem cells, and regenerative medicine research portal. PLOS ONE 8(7):e66629 http://www.plosone.org

Eldredge N, Gould JS (1972) Punctuated Equilibria: An alternative to phyletic gradualism. In: Schopf TJM (ed) Models in paleobiology. Freeman, Cooper, San Francisco, pp 85-115

Elsheikh M (2010) Towards a new physical theory of biotic evolution and development. Ecol Model 221:1108-1118

Elsheikh M (2014). Discovery of the life-organizing principle-in search of the fundamental laws of life. iUniverse LLC, Bloomington, IN.USA. 2014

Elsheikh M (2015) In Search of Quantum Information Biology. Adv Syst Biol 4(1):201. http://researchpub.org/journal/asb/archives. html

Goodwin BC (1985) What are the causes of morphogenesis? BioEssays 3:32-36

Gould SJ (1982) DARWINISM and the Expansion of Evolutionary Theory. Science 21:380-387

Grandpierre A (2007) Biological extension of the action principle. NeuroQuantology 4:346-367

Jorgensen SE (2006) Towards a Thermodynamics of Biological Systems. Int J Ecodyn 1:1-19

Jorgensen SE (2007) Evolution and exergy. Ecol Model. doi:10.1016/ j.ecolmodel.2006.12.035

Jorgensen SE (2012) Introduction to systems ecology. CRC, Boca Raton, $\mathrm{p} 137$

Jorgensen SE (2015) New Method to calculate the work energy of information an organism. Ecol Model 295:18-20

Kauffman S (1995) At home in the universe. Oxford University Press, Oxford

Lloyd S (2002) Computational capacity of the universe. Phys Rev Lett 88:237901-237908

Markov. A., Anisimov, V. Korotayev A. 2010. Relationship Between Genome Size and Organismal Complexity in the Lineage Leading from Prokaryotes to Mammals. ISSN 0031_0301, Paleontological Journal, 2010, Vol. 44, No. 4, pp. 363-373

Mandelbrot B (1982) The fractal geometry of nature. Freeman Co., San Francisco

McClendon JH (1980) The Evolution of the Chemical Isotopes as and Analog of Biological Evolution. J Theor Biol 87:113-128
Meyer SC (2004) The origin of biological information and the higher taxonomic categories. Proc Biol Soc Wash 117(2):213-239

Minorsky N (1962) Nonlinear Oscillations In: Robert E (ed). Krieger Publishing Company, Malabar, pp 71-73

Nicolis G, Prigogine I (1977) Self organization in non equilibrium systems: From dissipative structures to order through fluctuations. Wiley-Interscience Publication/John Wiley \& Sons, New York/London

Okasha S (2005) Multilevel selection and the major transitions in evolution. Philos Sci 72(5):1013-1025

Pareto V (1897) Cours d'economie politique, republished as Manual of political economy. Kelley Publishers, Augustus M

Pattee H (1968) The physical basis of coding and reliability. In: Waddington $\mathrm{H}$ (ed) Towards a theoretical biology. Edingburgh University Press, Edingburgh

Perez Jean-Claude (2010) Codon populations in single-stranded whole human genome DNA are fractal and fine-tuned by the golden ratio 1.16. Interdesci Sci Corput Life 2:228-240

Rosindell J (2012) A Fractal Explorer for the Tree of Life. PLOS Biol 10(10): e1001406. www.plosbiology.org

Sharov A (2006) Genome increase as a clock for the origin and evolution of life. Biol Direct 2006(1):17. doi:10.1186/17456150-1-17

Thomson KS (1992) Macroevolution: the morphological problem. Am Zool 32:106-112

Truman R (1999) The problem of information for the theory of evolution: Has Dawkins really solved it? http://www.trueorigin. org/dawkinfo.asp

Waddington CH (1968a) Does Evolution Depend on Random Search? In: Waddington $\mathrm{CH}$ (ed) Towards a Theoretical Biology. The Kynock Press, Birmingham

Waddington CH (1968b) The Basic Ideas of Biology. In: Waddington $\mathrm{CH}$ (ed) Towards a theoretical biology1: Prolegomena. Edinburgh University Press, Edinburgh

Waddington CH (1968c) Does evolution depend on random Search? In: Waddington $\mathrm{CH}$ (ed) Towards a theoretical biology. The Keynock Press, Birmingham

Whyte LL (1965) Internal factors in evolution. Tavistock Publications, London

Wilson DS, Wilson EO (2007) Rethinking the Theoretical Foundation of Sociobiology. Q Rev Biol 32(4):327

Winter D (2012) Golden ratio constructive wave interference. http:// www.goldenmean.info/fractalfield/

Winter D http://www.fractalfield.com/mathematicsoffusion/

Yockey HP (1992) Information theory and molecular biology. CambridgeUniv Press, Cambridge, pp 255-257 